\title{
Model Predictive Control for operator-in-the-loop overhead cranes
}

\author{
Marco Giacomelli ${ }^{1}$, Marco Faroni ${ }^{1}$, Domenico Gorni ${ }^{1}$, Alberto Marini ${ }^{1}$, Luca Simoni ${ }^{1}$, and Antonio Visioli ${ }^{1}$ \\ ${ }^{1}$ Dipartimento di Ingegneria Meccanica e Industriale, Università degli Studi di Brescia, Italy \\ \{m.giacomelli009, m.faroni003, domenico.gorni, alberto.marini, luca.simoni, antonio.visioli\}@unibs.it
}

\begin{abstract}
In this paper, a Model Predictive Control approach for the velocity control of operator-in-the loop overhead cranes is proposed. The operator can select the maximum position overshoot as a tuning parameter for the method. Simulations provide a comparison between the proposed method and the well known Zero Vibration input shaping technique, showing its effectiveness in controlling the payload oscillations.
\end{abstract}

Index Terms-Overhead crane, MPC, oscillation reduction, operator-in-the-loop.

\section{INTRODUCTION}

Overhead cranes are the most diffused material handling systems in industry, due to their simple design. Nonetheless, cranes are underactuated oscillatory systems, and their control is non-trivial. The control of overhead cranes has raised increasing interest in both research and industrial fields in the last thirty years [1], [2], aiming at the reduction of positioning errors and travelling time [3], [4].

In particular, manual control represent a major issue, as the presence of a strongly underdamped dynamics decreases the accuracy and increases positioning time, while the uncontrolled oscillation of heavy loads poses a serious safety hazard.

A number of different techniques is present in the literature, and they can be divided in two main categories: open and closed-loop techniques. Open-loop control techniques are simpler, as they do not require additional sensors for the measurement of the states of the system, relying only on its model, and they are therefore easily implementable in off-theshelf industrial cranes. For the control of residual oscillations of overhead cranes optimal control has been proposed in [5], [6]. Input shaping technique is the most widely used openloop technique for the residual oscillation control of industrial overhead crane, due to its easy implementation and its intuitive and easy-to-measure parameters, namely only the oscillation period of the crane and its damping coefficient, therefore not requiring a mathematical model of the system [7]-[9]. Input-output inversion has also been proposed for the open loop control of overhead cranes [10]-[12]. This technique relies on the definition of a desired output trajectory and on the inversion of the transfer function of the model to obtain a suitable input that guaratees null residual oscillation. Both input shaping and input-output inversion techniques have also been proposed for cranes which exhibit double-pendulum dynamics [13], [14].
Closed-loop techniques are more difficult to implement in off-the-shelf industrial overhead cranes, due to the fact that closed-loop techniques require for the measurement of the states (or the output) of the system to be controlled, while industrial cranes lack of the sensors to measure those states. The decreasing cost of sensors in general and the increased robustness of closed-loop technique with respect to open-loop techniques justify the use of closed-loop control techniques in the control of industrial overhead crane [15].

Linear and nonlinear techniques have been proposed for the anti-swing control of gantry cranes [16], [17]. Moreover, different control strategies have been investigated over the years. For example, in [18]-[21] adaptive control is applied to the problem, while in [22]-[24] fuzzy control techniques have been proposed.

The increasing computational power of modern industrial controllers has also made possible the application of Model Predictive Control (MPC) in fast dynamics applications (e.g., robotics and mechatronics). Due to its capability of respecting and exploit the imposed limits, MPC has become one of the most popular control algorithms for the control of multivariable systems. The use of MPC in the control of gantry and overhead cranes is motivated by its ability in dealing with constraints of multivariable systems, allowing the explicit limitation of the maximum sway angle, and its robustness against parametric uncertainties; MPC has been proven to be a competitive solution for the control of cranes with respect to standard open-loop and feedback control [25]. Moreover, a solution for computational infeasibility of MPC on fast dynamics systems like cranes has been proposed in [26] limiting the maximum iterations of the optimization solver obtaining a suboptimal model predictive control.

Most of the research on the application of MPC controllers to gantry cranes has focused on the position control and the sway angle reduction [27], [28]. Some of the proposed MPC applications are based on the tracking of an offline computed trajectory, as in [29] where nonlinear MPC is used for the tracking of trajectories in a 3D environment, using the length of the rope as a gain scheduling parameter. In [30], [31] the MPC algorithm is used to optimize the energy consumption for a point-to-point motion, tracking a trajectory obtained with an offline optimization minimizing a cost function with a weight on the consumed energy. In [32] the use of MPC for trajectory tracking of a $3 \mathrm{D}$ overhead crane is proposed, using a reduced- 
order decoupled linear system for the design. Other proposed techniques do not follow a predetermined trajectory, but only require the final position. A multi-criteria cost function has been proposed in [33] for a minimum transfer time weighting also the sway angle, but constraints are ignored during the optimization procedure. In [34] the problem of constrained control of an overhead crane has been transformed into an unconstrained optimization problem, while in [35] the bounds on the swing angle have been converted into some constraints on the control input. In [36] a fast Nonlinear MPC technique based on automatic code generation has been proposed.

While position control schemes are appropriated for completely automatic cranes, the majority of the industrial cranes is manually controlled by operators. For this case scenario, a position control like the ones proposed in the aforementioned publications in not implementable, and a velocity control must be used. As a matter of fact, when the operator presses the movement button, it is not possible to define an end position or a trajectory to be followed, but it is desirable that the payload reaches the desired velocity in the minimum time and it stops without residual oscillations and with an acceptable position overshoot when the operator releases the button.

Input shaping techniques have been proposed for the anti-sway control of operator actuated cranes [37]-[39]. In [40] open loop techniques were shown to perform better than closed-loop PD control, with less energy consumption and low average completion time. The use of closed-loop techniques for the control of operator-in-the-loop overhead cranes is still an open issue [41].

However, the implementation of efficient closed-loop techniques for the velocity control of overhead cranes has a strong relevance from a practical perspective, as it would bring an increased robustness in the operations with respect to the now diffused open-loop techniques.

To the best of the authors knowledge, MPC has never been applied to velocity control of overhead crane systems, limiting the application of MPC to fully automated systems. For this reason, this paper proposes an MPC approach to velocity control of a gantry crane. The proposed strategy is based on the velocity control of the payload: when the operator presses the movement button, a step with proper amplitude is set as new reference for the payload velocity; when the operator releases the button, the reference is set to zero. A term weighting the sway angular velocity is introduced in the cost function to reduce oscillatory behaviors. As the gantry crane is modeled as a single pendulum and linearized by means of smallangle approximations, linear MPC can be used. Moreover, constraints on the maximum force and velocity provided by the actuated cart are included in the formulation. The paper also provides a simple procedure for the choice of a proper value for the velocity step given to the payload. Such procedure allows the choice of the velocity step amplitude based on the maximum position overshoot expected at the end of the stop phase (i.e., when the operator releases the button). Note that such overshoot limitation could not be straightforwardly implemented in the MPC framework as a constraint on the

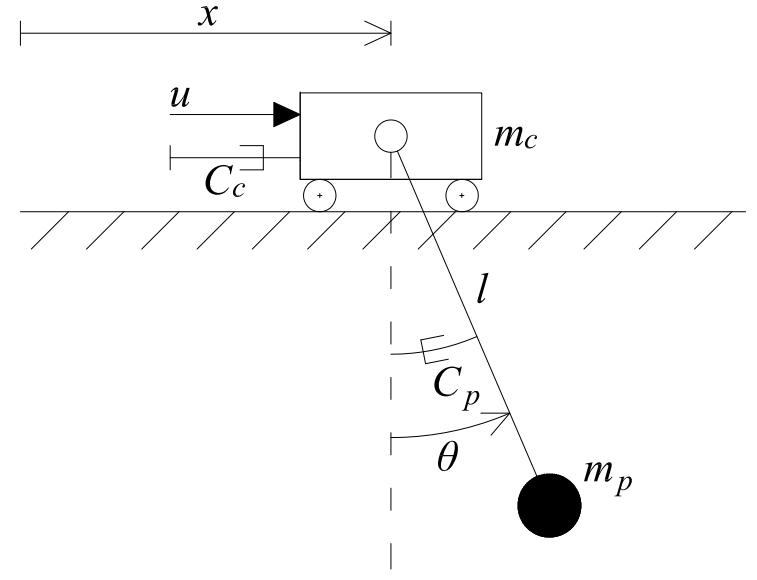

Fig. 1: Scheme of a overhead crane seen as a simple pendulum connected to a sliding cart. Figure adapted from [42].

output position. As a matter of fact, naively imposing such constraint in the optimization would not ensure the feasibility of the problem. Such approach would need an invariance analysis of the system, in order to determine the state region within which such constraints can be satisfied. Moreover, such analysis should be repeated whenever the maximum allowed overshoot is modified. The procedure proposed in the paper permits to avoid such complications, giving an easy way to satisfy the maximum overshoot limits just by choosing a proper amplitude of the velocity step.

The method is tested via simulation on a nonlinear model of the crane, with uncertainties in the parameters, and the results are finally compared with Zero Vibration (ZV) input shaping, the most widely used oscillation reduction algorithm in industry.

The paper is organized as follows. In II the model of a gantry crane is presented, including in its dynamics the contribute of friction. In III the proposed method is outlined in detail, while in IV it is shown how the velocity reference is defined taking into account the maximum position overshoot defined by the operator. In $\mathrm{V}$ the results obtained from simulations are discussed, comparing the performance of the proposed method with an input-shaping anti sway control algorithm in terms of robustness. VI conclude the paper.

\section{Modelling OF GANTRY CRAne SyStem}

As a first approximation, an overhead crane can be schematized as a single pendulum and a moving cart, as shown in Figure 1. The symbols refer to:

$u$ : force applied to the cart;

$m_{C}$ : mass of the cart;

$C_{C}$ : viscous friction coefficient of the cart;

$\theta$ : angle between the vertical and the cable (between cart and payload);

$C_{p}$ : viscous friction coefficient of the cable;

$l \quad$ : length of the cable;

$m_{p} \quad$ : mass of the payload. 
By considering the rope as an inflexible rod, with a negligible mass with respect to the mass of the payload, the equations of the overhead crane dynamics can be derived using Lagrangian formalism, obtaining

$$
\left\{\begin{array}{l}
\left(m_{p}+m_{C}\right) \ddot{x}+m_{p} l\left(\ddot{\theta} \cos \theta-\dot{\theta}^{2} \sin \theta\right)=u-C_{C} \dot{x} \\
m_{p} \ddot{x} \cos \theta+m_{p} l \ddot{\theta}+m_{p} g \sin \theta=-\frac{C_{p}}{l} \dot{\theta}
\end{array}\right.
$$

The model can now be linearized around its sole stable equilibrium point, that is, $\theta=0, \dot{\theta}=0$. For small angles, (1) can be approximated as

$$
\left\{\begin{array}{l}
\left(m_{p}+m_{C}\right) \ddot{x}+m_{p} l \ddot{\theta}+C_{C} \dot{x}=u \\
m_{p} \ddot{x}+m_{p} l \ddot{\theta}+\frac{C_{p}}{l} \dot{\theta}+m_{p} g \theta=0
\end{array}\right.
$$

Define the state vector as $x_{s s}:=(x, \dot{x}, \theta, \dot{\theta})^{T}$ and consider four outputs:

(i) the payload position $y_{0}=x+l \sin \theta$;

(ii) the payload velocity $y_{1}=\dot{x}+l \dot{\theta} \cos \theta$;

(iii) the sway angular velocity $y_{2}=\dot{\theta}$;

(iv) the cart velocity $y_{3}=\dot{x}$.

By defining the output vector as $y=\left(y_{0}, y_{1}, y_{2}, y_{3}\right)^{T}$ and by using the approximation for small angles, the model can be rearranged in state-space formulation as

$$
\left\{\begin{array}{l}
\dot{x}_{s s}(t)=A x_{s s}(t)+B u(t) \\
y(t)=C x_{s s}(t)
\end{array}\right.
$$

where

$$
\begin{aligned}
A & =\left(\begin{array}{cccc}
0 & 1 & 0 & 0 \\
0 & -\frac{C_{C}}{m_{C}} & \frac{g m_{p}}{m_{C}} & \frac{C_{p}}{m_{C}} \\
0 & 0 & 0 & 1 \\
0 & \frac{C_{C}}{l m_{C}} & -\frac{g\left(m_{p}+m_{C}\right)}{l m_{C}} & -\frac{C_{p}\left(m_{p}+m_{C}\right)}{l^{2} m_{p} m_{C}}
\end{array}\right) \\
B & =\left(\begin{array}{cccc}
0 & \frac{1}{m_{C}} & 0 & \left.-\frac{1}{l m_{C}}\right)^{T}
\end{array}\right. \\
C & =\left(\begin{array}{llll}
C_{0}^{T} & C_{1}^{T} & C_{2}^{T} & \left.C_{3}^{T}\right)^{T}
\end{array}\right.
\end{aligned}
$$

with

$$
\begin{aligned}
C_{0} & =\left(\begin{array}{llll}
1 & 0 & l & 0
\end{array}\right) \\
C_{1} & =\left(\begin{array}{llll}
0 & 1 & 0 & l
\end{array}\right) \\
C_{2} & =\left(\begin{array}{llll}
0 & 0 & 0 & 1
\end{array}\right) \\
C_{3} & =\left(\begin{array}{llll}
0 & 1 & 0 & 0
\end{array}\right) .
\end{aligned}
$$

Moreover, a real overhead crane is usually subject to hardware limitations, nominally the maximum and minimum force that can be applied to the card $u_{\max }$ and $u_{\min }$, and the maximum and minimum velocity of the cart $\dot{x}_{\max }$ and $\dot{x}_{\min }$.

\section{PRoposed CONTROL ALGORITHM}

In manual control, the operator commands the movement of the crane by pressing a button: if the button is pressed, the payload should move forward; if the button is not pressed, the payload should remain still. For the purposes of the present work, this is expressed by defining the reference for the payload velocity as:

$$
w_{1}:= \begin{cases}\alpha & \text { if button is pressed } \\ 0 & \text { if button is not pressed }\end{cases}
$$

where $\alpha \in \mathbb{R}^{+}$is the amplitude of the velocity step. The choice of a proper value of $\alpha$ will be discussed in Section IV.

The application of classic MPC to the velocity control of the payload is straightforward. To this purpose, consider a predictive horizon $p \in \mathbb{N}$ and a control horizon $c \in \mathbb{N}$, which respectively represent the number of future sampling periods of the output prediction and the number of future control actions taken into account by the prediction. The predictive equations of the outputs at time $k$ can be written in the form

$$
\begin{aligned}
& Y_{1}=F_{1} x(k)+G_{1} U \\
& Y_{2}=F_{2} x(k)+G_{2} U \\
& Y_{3}=F_{3} x(k)+G_{3} U
\end{aligned}
$$

where $Y_{1}:=\left(y_{1}(k+1), \ldots, y_{1}(k+p)\right)^{T} \in \mathbb{R}^{p}$ (and likewise for $Y_{2}$ and $\left.Y_{3}\right), U:=(u(k), \ldots, u(k+c-1))^{T} \in \mathbb{R}^{c}, x(k) \in \mathbb{R}^{4}$ is the current state vector, and $F_{1}, F_{2}, F_{3} \in \mathbb{R}^{p \times 4}$ and $G_{1}, G_{2}, G 3 \in$ $\mathbb{R}^{p \times c}$ are predictive matrices calculated from $A, B$, and $C$ as in [43]-[45].

The Optimal Control Problem (OCP) to be solved at each cycle by the MPC controller is defined as a Quadratic Program (QP) that minimizes the deviation between the predicted outputs and their desired references. In manual control, the controller should minimize the deviation between the payload velocity and the reference $w_{1}$. Moreover, the sway angular velocity should be minimized as well to reduced undesired oscillations. Thus, the OCP is defined as the following optimization problem:

$$
\begin{array}{ll}
\operatorname{minimize} & \left\|Y_{1}-w_{1}\right\|^{2}+\lambda_{2}\left\|Y_{2}\right\|^{2}+\lambda_{u}\|U\|^{2} \\
\text { subject to } & \dot{x}_{\min } \leq Y_{3} \leq \dot{x}_{\max } \\
& u_{\min } \leq U \leq u_{\max }
\end{array}
$$

where $\lambda_{2}, \lambda_{u} \in \mathbb{R}^{+}$are weighting factors for the sway angular velocity and the control effort minimization respectively. As the controller primal goal consists in tracking the payload velocity reference, $\lambda_{2}$ and $\lambda_{u}$ should be small enough. Note that the reference value $w_{1}$ along the predictive horizon is kept equal to its value at time $k$ along the whole predictive horizon, as the time instant at which the operator will change the button state is not predictable by the algorithm. 
Problem (11) can be written in standard quadratic form and with respect to the control vector $U$ as follows:

$$
\begin{array}{ll}
\operatorname{minimize} & \frac{1}{2} U^{T} M U+N^{T} U \\
\text { subject to } & E U \leq D \\
& u_{\min } \leq U \leq u_{\max }
\end{array}
$$

where:

$$
\begin{aligned}
M & :=G_{1}^{T} G_{1}+\lambda_{2} G_{2}^{T} G_{2}+\lambda_{u} I_{c} \\
N & :=G_{1}^{T}\left(F_{1} x(k)-w_{1}\right)+\lambda_{2} G_{2}^{T} F_{2} x(k) \\
E & :=\left(G_{3}^{T},-G_{3}^{T}\right)^{T} \\
D & :=\left(\dot{x}_{\max }-F_{3} x(k),-\dot{x}_{\min }+F_{3} x(k)\right)^{T}
\end{aligned}
$$

where $I_{c}$ denotes the identity matrix. As typical of MPC, such OCP has to be solved at each iteration. The first element of $U$ is therefore applied to the system and the procedure is repeated by applying a receiding horizon concept.

\section{CHOICE OF THE VElocity STEP AMPLITUdE}

The position overshoot of the payload is the longitudinal distance covered by the payload since when the operator releases the button. It is clear that such overshoot depends on the system state at the time of release. Moreover, it depends on the control law adopted by the controller and on the system constraints. We want to determine the maximum amplitude of the velocity step $\alpha$ such that the maximum position overshoot $\Delta x$ does not exceed a given value $\Delta x_{\max }$. This can be written as an optimization problem as follows:

$$
\begin{array}{ll}
\text { maximize } & \alpha \\
\text { subject to } & \Delta x(t) \leq \Delta x_{\max } \forall t \geq t_{B}
\end{array}
$$

where $t_{B}$ is the time instant when the operator releases the jog button.

To solve (17) numerically, assume that: (i) the predictive horizon $p$ is long enough to take into account the maximum position overshoot, (ii) when the operator releases the button, the system is at steady-state, that is, $x\left(t_{B}\right)=(0, \alpha, 0,0)^{\prime}=: x_{0}$. The predicted overshoot can be therefore calculated as:

$$
\Delta X=F_{0} x_{0}+G_{0} U_{0}
$$

where $\Delta X:=(\Delta X(k+1), \ldots, \Delta X(k+p))^{T}, F_{0} \in \mathbb{R}^{p \times 4}$ and $G_{0} \in$ $\mathbb{R}^{p \times c}$ can be computed from $A, B, C_{0}$, similarly to the predictive matrices in (8), and $U_{0}$ is calculated as the solution of (12), with $x(k)=x_{0}$. Thus, problem (17) becomes:

$$
\begin{aligned}
& \text { maximize } \alpha \\
& \text { subject to } F_{0} x_{0}+G_{0} U_{0} \leq \Delta x_{\max } \\
& x_{0}=(0, \alpha, 0,0)^{T}
\end{aligned}
$$

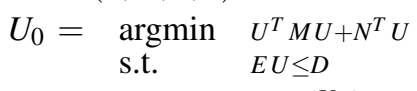

$$
\begin{aligned}
& u_{\min } \leq U \leq u_{\min }
\end{aligned}
$$

Problem (19) can be easily solved by using, for example, the secant method to find the root of the equation $f(\alpha)=0$, where $f:\left[0, \dot{x}_{\max }\right] \subseteq \mathbb{R} \rightarrow \mathbb{R}, \alpha \mapsto \max (\Delta X(\alpha))-\Delta x_{\max }$.

\section{Simulations RESUlts}

The proposed methodology has been tested on a nonlinear model of an overhead crane in Simulink, built inside the Simscape Multibody environment [46].

We expect the proposed MPC technique to be more robust than an open loop technique like input shaping with respect to nonlinearities and errors in the model parameters. For the purposes of comparison, in the simulation environment an input shaping technique has also been implemented. We choose to implement the easiest of the input shaping techniques, the Zero-Vibration (ZV), as it is the most widely diffused technique for the control of residual oscillations in industry [41]. To prove this, two different simulation scenarios were discussed: in the first one, perfect knowledge og the model is assumed, thus the control algorithms are devised by using the exact parameters of the controlled system. Secondly, a mismatch in the model parameters is introduced to test the robustness of the proposed method. The parameters used for the model for control purposes are listed in Table I, in the first scenario, the simulation model has the same parameters of the control model. On the second scenario, the simulation model has the parameters of the third column of Table I, while the control model keeps the parameter of the second column. The difference in the parameters values underlines the robustness of the tested methods with respect to errors in the modellization.

The limits for the simulated overhead crane imposed in (11)

TABLE I: Parameters of the model used in simulations.

\begin{tabular}{|l|l|l|}
\hline \multicolumn{3}{|c|}{ Model data } \\
\hline Parameter & Exact parameters & parameters with errors \\
\hline$m_{C}$ & $38[\mathrm{~kg}]$ & $45[\mathrm{~kg}]$ \\
$C_{C}$ & $20\left[\frac{\mathrm{Ns}}{\mathrm{m}}\right]$ & $25\left[\frac{\mathrm{Ns}}{\mathrm{m}}\right]$ \\
$C_{p}$ & $0.1\left[\frac{\mathrm{Nms}}{\mathrm{rgd}}\right]$ & $0.2\left[\frac{\mathrm{Nms}}{\mathrm{rgd}}\right]$ \\
$l$ & $1.5[\mathrm{~m}]$ & $1.3[\mathrm{~m}]$ \\
$m_{p}$ & $10[\mathrm{~kg}]$ & $12[\mathrm{~kg}]$ \\
\hline
\end{tabular}

are set to

$$
\dot{x}_{\max }=-\dot{x}_{\min }=1.2[\mathrm{~m} / \mathrm{s}] \quad u_{\max }=-u_{\min }=150[\mathrm{~N}]
$$

while the weighting parameters in (11) are set to

$$
\lambda_{2}=5.0 \cdot 10^{-4} \quad \lambda_{u}=1.0 \cdot 10^{-6} .
$$

The values in (21) have been tuned by trial and error method. The maximum payload overshoot in (17) is set to

$$
\Delta x_{\max }=0.5[m]
$$

, from which $\alpha$ results to be equal to $\alpha=0.9850$ by solving the optimization problem (19). The sampling time has been set to $T_{s}=0.01[s]$; the predictive horizon has been set to $p=100$, while the control horizon to $c=50$.

During the simulation, at time $t=1.0[s]$ a velocity step $\alpha$ is given to the system. At time $t=7.0[s]$ the velocity reference is set to zero. The velocity reference is shown in Figure 10. 


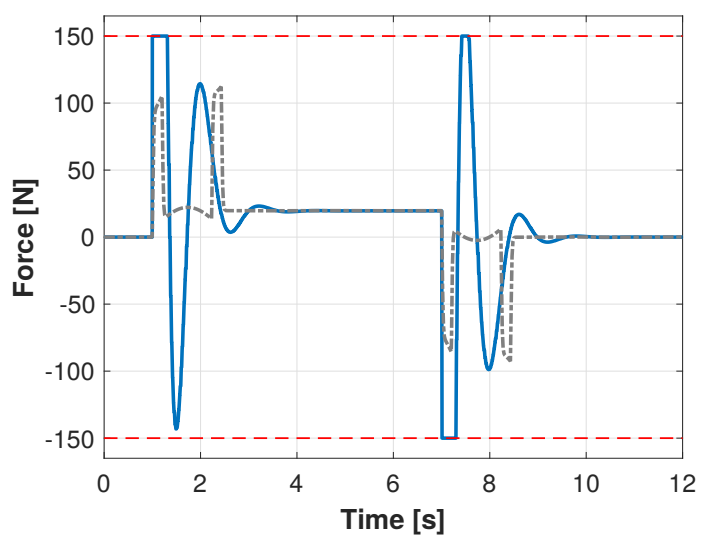

Fig. 2: Force acting on the cart. Solid blue: proposed method. Dashed-dotted grey: ZV input shaping. Dashed red: cart force limits.

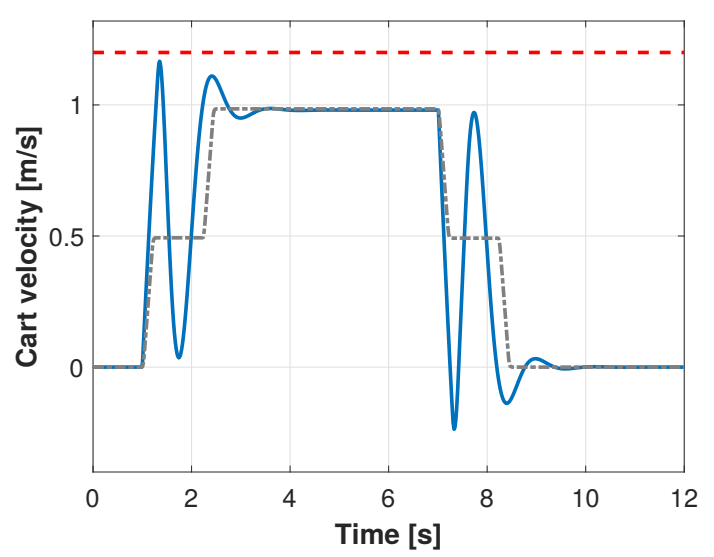

Fig. 3: Velocity of the cart during the control, for both MPC (blue solid line) and input-shaping (grey dash-dot line) in the case of exact model parameters. The dashed red line represents the velocity limit imposed to the cart.

\section{A. Nominal case}

First, the MPC approach has been tested by controlling a system with exact parameter values, comparing the results with the ones obtained using ZV input-shaping technique. In Figure 2 the force acting on the cart is shown. It can be seen that the MPC approach respects and fully exploits the imposed limits on the force. Figure 3 shows that the velocity of the cart is successfully contained within its limits.

In Figure 4 the payload velocity is shown for both input shaping and model predictive control. Due to the aggressive tuning of the values in (21) oscillations are present in the velocity response when using MPC, but they are rapidly compensated. On the other hand, with $\mathrm{ZV}$ input shaping virtually no oscillations are present (only small residual oscillations due to non-linearities). The evolution of the sway angle is shown in Figure 5. In Figure 6 the position of the payload shows no significant difference in the sense of total displacement. In

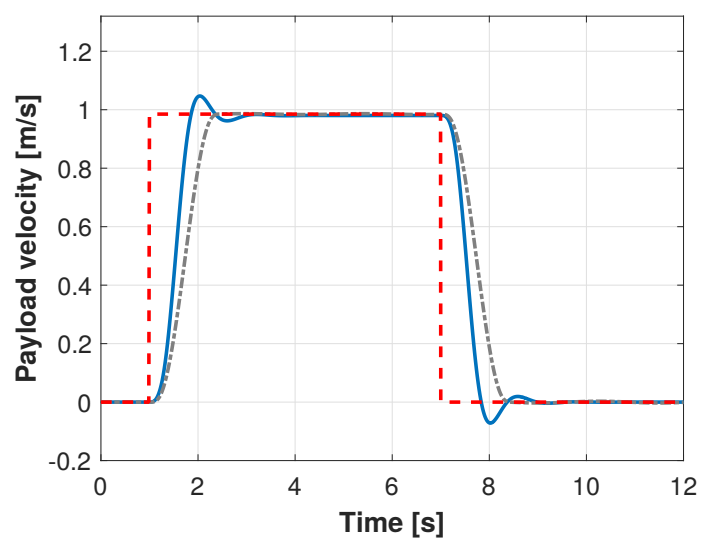

Fig. 4: Velocity of the payload with MPC (blue solid line) and input-shaping (grey dash-dot line). In red dashed line the velocity reference.

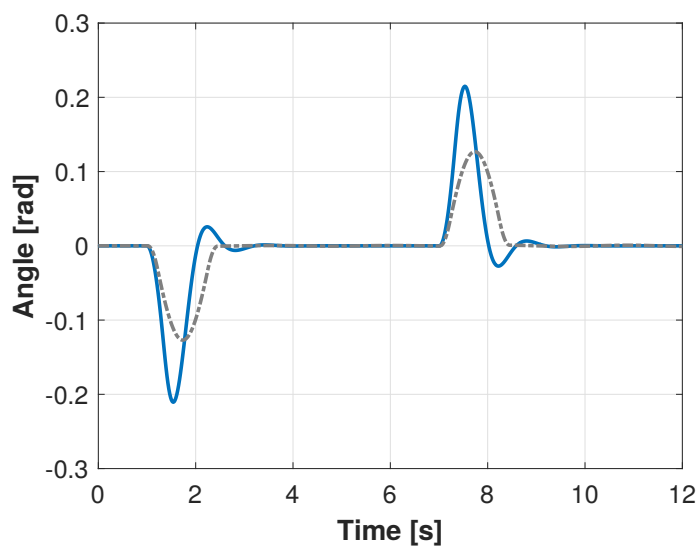

Fig. 5: Swing angle of the payload in the case of exact model parameters, for both MPC (blue solid line) and input-shaping (grey dash-dot line).

Figure 7 the position overshoot is shown. The overshoot is calculated as the integral of the velocity of the payload after the operator has released the button, that is after $t=7[\mathrm{~s}]$ in our case. It is possible to note that the imposed limit (22) is slightly exceeded due to the nonlinearities in the simulation model; the aggressiveness of the tuning of MPC guarantees a reduced position overshoot with respect to the $\mathrm{ZV}$ case.

\section{B. Errors in model parameters}

The parameters of the simulated model have then been changed according to the values in the third column of Table I in order to test the robustness of the proposed method. In Figure 8 and in Figure 9 the force acting on the cart and the velocity of the cart are shown, respectively. It can be seen how, also in the case of errors in the model parameters, both limits on force and velocity are respected.

In Figure 10 the payload velocity is shown for both input shaping and model predictive control in the case of errors in model parameters. It can be seen that, due to the errors 


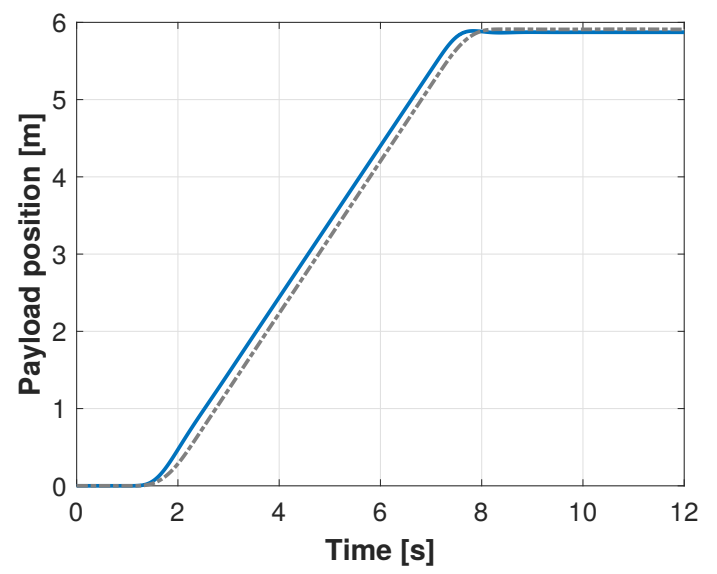

Fig. 6: Position of the payload in the case exact model parameters. Solid blue: proposed method. Dashed-dotted grey: ZV input shaping.

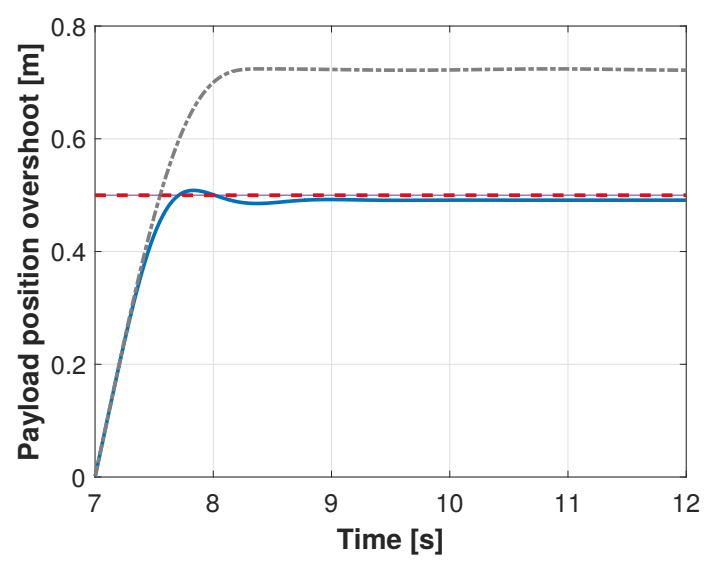

Fig. 7: Position overshoot of the payload in the case exact model parameters. Solid blue: proposed method. Dasheddotted grey: $\mathrm{ZV}$ input shaping. The red dashed line is the imposed limit.

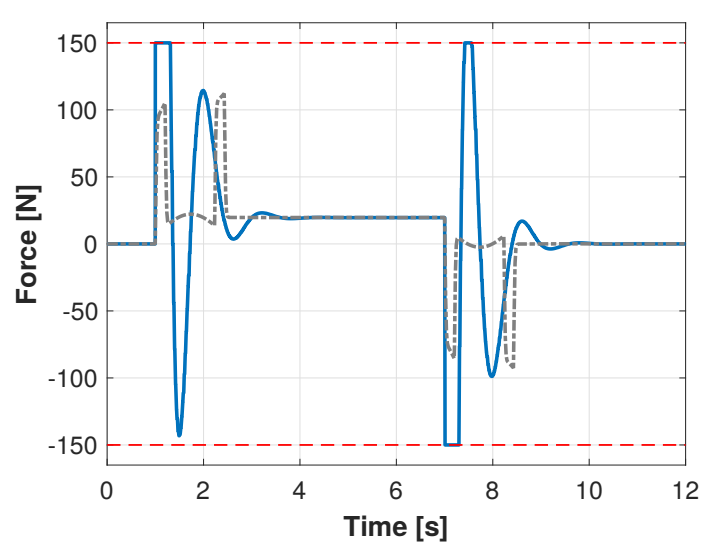

Fig. 8: Force acting on the cart in the case of errors in the model parameters. Solid blue: proposed method. Dasheddotted grey: ZV input shaping. Dashed red: cart force limits.

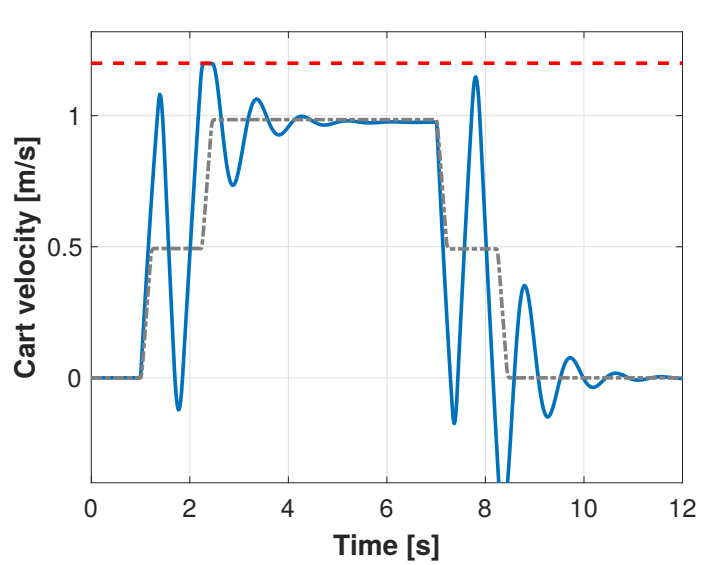

Fig. 9: Velocity of the cart during the control, for both MPC (blue solid line) and input-shaping (grey dash-dot line) in the case of errors in the model parameters. The dashed red line represents the velocity limit imposed to the cart.

introduced in the parameters of the system, oscillations are present for both control strategies. Nonetheless, the MPC control rapidly reduces the oscillations, while the oscillations are not compensated with input shaping techniques, leading to the presence of residual oscillations. The reduction of the oscillation can be observed in Figure 11: the MPC strategy brings the sway angle to zero. In Figure 12 the position of the payload shows no significant difference in the sense of total displacement between the two different techniques. In Figure 13 the position overshoot of the payload is shown for both techniques, showing that, despite uncertainties and nonlinearities, the position overshoot slightly exceeds the offline imposed limit, showing no significant deterioration with respect to the exact parameters case in Figure 7. Moreover, the position overshoot with the proposed technique is significantly reduced with respect to the one introduced by the use of $\mathrm{ZV}$ input-shaping techniques.

\section{CONCLUSIONS}

In this paper, an MPC approach for the velocity control of the payload of an overhead crane has been presented.

The set-point velocity is calculated online, depending on the maximum position overshoot of the payload desired, which therefore is a tunable parameter and can be set depending on the application. The presented method has been proven to be effective in controlling the oscillations of the payload. Unlike standard open loop techniques like ZV input-shaping, the presented method for the velocity control of the payload has also proved to be robust with respect to errors in the model parameters and nonlinearities.

Future developments of the proposed technique will be its implementation on an industrial overhead crane, in order to prove the feasibility of the technique with off-the-shelf industrial components. Also, an MPC based technique should be implemented for the velocity control of overhead cranes taking into account explicitly the hoisting maneuver. 


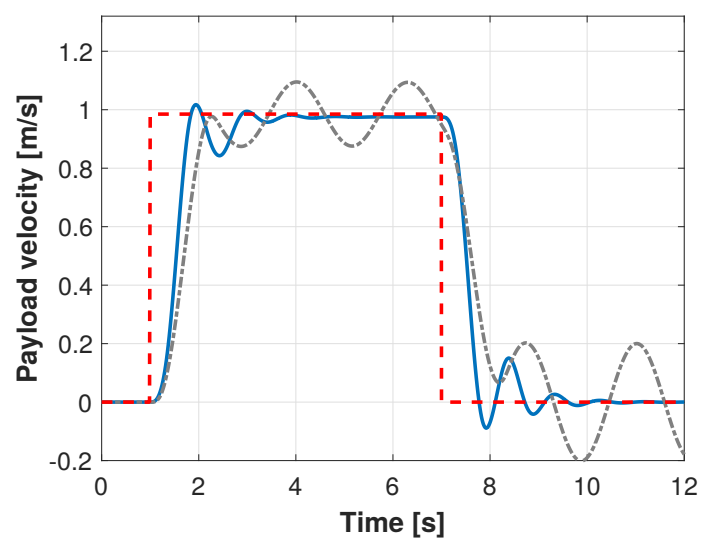

Fig. 10: Velocity of the payload with MPC (blue solid line) and input-shaping (grey dash-dot line) control in the case of errors in the model parameters. In red dashed line the velocity reference.

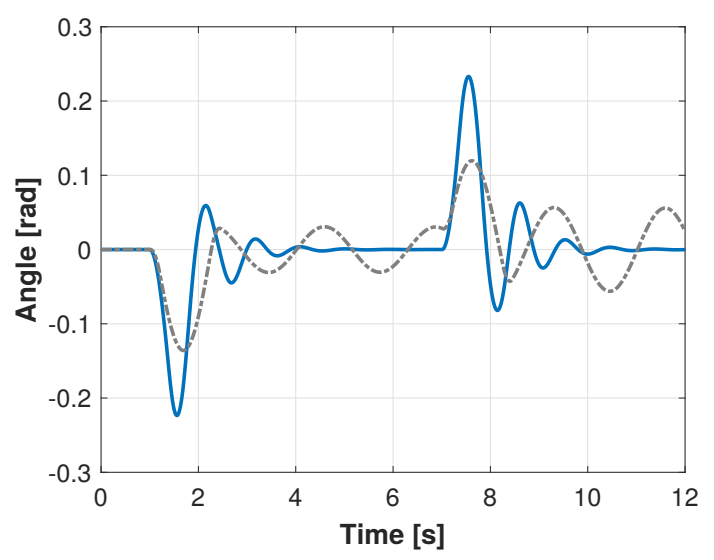

Fig. 11: Swing angle of the payload in the case of errors in the model parameters, for both MPC (blue solid line) and inputshaping (grey dash-dot line).

\section{ACKNOWLEDGEMENTS}

This work has been developed within the European Union H2020 program ECSEL-2016-1 under grant agreement $n$. 737453 (I-MECH).

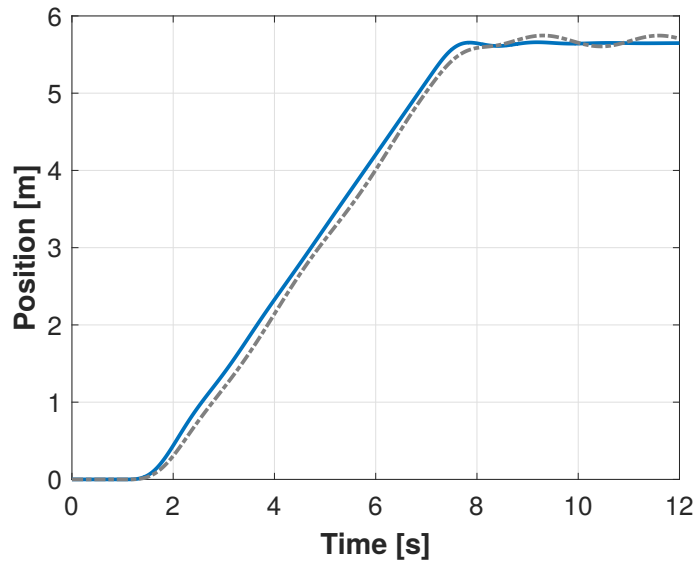

Fig. 12: Position of the payload in the case of errors in the model parameters. Solid blue: proposed method. Dasheddotted grey: ZV input shaping.

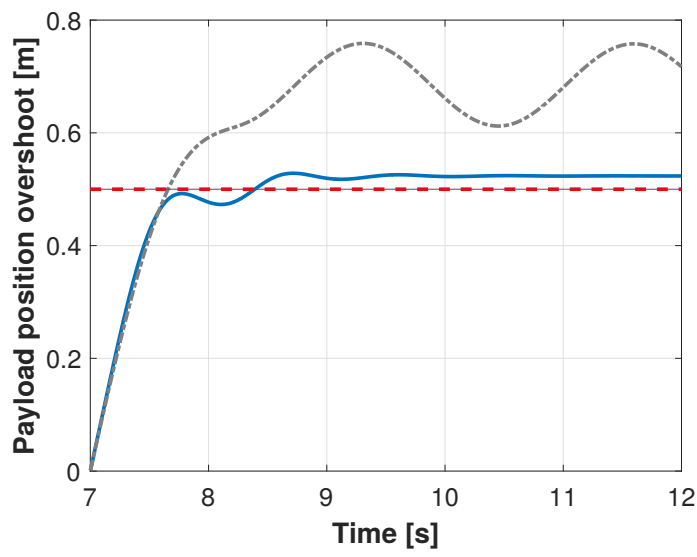

Fig. 13: Position overshoot of the payload in the case of errors in the model parameters. The red dashed line is the imposed limit.

\section{REFERENCES}

[1] E. M. Abdel-Rahman, A. H. Nayfeh, and Z. N. Masoud, "Dynamics and control of cranes: A review," Modal Analysis, vol. 9, no. 7, pp. 863-908, 2003.

[2] P. Hyla, "The crane control systems: A survey," in Prooceedings of the IEEE International Conference on Methods and Models in Automation and Robotics, 2012, pp. 505-509.

[3] X. Zhang, Y. Fang, and N. Sun, "Minimum-time trajectory planning for underactuated overhead crane systems with state and control constraints," IEEE Transactions on Industrial Electronics, vol. 61, no. 12, pp. 6915-6925, 2014.

[4] J. Auernig and H. Troger, "Time optimal control of overhead cranes with hoisting of the load," Automatica, vol. 23, no. 4, pp. 437-447, 1987.

[5] J. A. Field, "The optimization of the performance of an ore bridge," Transactions of the Engineering Institute of Canada, vol. 5, no. 3, pp. 163-169, 1961.

[6] A. Al-Garni, K. Moustafa, and S. J. Nizami, "Optimal control of overhead cranes," Control Engineering Practice, vol. 3, no. 9, pp. 12771284, 1995.

[7] S. S. Gürleyük, Ö. Bahadir, Y. Türkkan, and H. ÜŞENTİ, "Improved three-step input shaping control of crane system," Constraints, vol. 6, p. 10,2008 
[8] N. C. Singer and W. P. Seering, "Preshaping command inputs to reduce system vibration," Journal of Dynamic Systems, Measurement, and Control, vol. 112, no. 1, pp. 76-82, 1990.

[9] W. E. Singhose, W. P. Seering, and N. C. Singer, "Shaping inputs to reduce vibration: a vector diagram approach," in Proceedings of the IEEE International Conference on Robotics and Automation, 1990, pp. 922-927.

[10] A. Piazzi and A. Visioli, "Minimum-time system-inversion-based motion planning for residual vibration reduction," IEEE/ASME Transactions on Mechatronics, vol. 5, no. 1, pp. 12-22, 2000.

[11] F. Padula, A. Visioli, D. Facchinetti, and A. Saleri, "A dynamic inversion approach for oscillation-free control of overhead cranes," in Proceedings of the IEEE International Conference on Emerging Technologies and Factory Automation, 2015, pp. 1-6.

[12] A. Piazzi and A. Visioli, "Optimal dynamic-inversion-based control of an overhead crane," IEE Proceedings-Control Theory and Applications, vol. 149, no. 5, pp. 405-411, 2002.

[13] W. E. Singhose, D. Kim, and M. Kenison, "Input shaping control of double-pendulum bridge crane oscillations," Journal of Dynamic Systems, Measurement, and Control, vol. 130, no. 3, 2008.

[14] M. Giacomelli, F. Padula, L. Simoni, and A. Visioli, "Simplified inputoutput inversion control of a double pendulum overhead crane for residual oscillations reduction," Submitted to Mechatronics.

[15] H. M. Omar and A. Nayfeh, "Anti-swing control of gantry and tower cranes using fuzzy and time-delayed feedback with friction compensation," Shock and Vibration, vol. 12, no. 2, pp. 73-89, 2005.

[16] H. Lee, S. Cho, and J. Cho, "A new anti-swing control of overhead cranes," IFAC Proceedings Volumes, vol. 30, no. 13, pp. 115-120, 1997.

[17] Y. Fang, W. Dixon, D. Dawson, and E. Zergeroglu, "Nonlinear coupling control laws for an underactuated overhead crane system," IEEE/ASME Transactions on Mechatronics, vol. 8, no. 3, pp. 418-423, 2003.

[18] J. Ackermann, "Parameter space design of robust control systems," IEEE Transactions on Automatic Control, vol. 25, no. 6, pp. 1058-1072, 1980.

[19] A. Marttinen, "Pole-placement control of a pilot gantry," in Proceedings of the American Control Conference, 1989, pp. 2824-2826.

[20] R. Salminen, A. Marttinen, and J. Virkkunen, "Adaptive pole placement control of a pilot crane," IFAC Proceedings Volumes, vol. 23, no. 8, pp. 313-318, 1990

[21] J. M. Virkkunen and A. T. Marttinen, "Computer control of a loading bridge," in Proceedings of the International Conference on Control, 1988, pp. 484-488.

[22] H. Oshima, S. Yasunobu, and S. Sekino, "Automatic train operation system based on predictive fuzzy control," in Artificial Intelligence for Industrial Applications, Proceedings of the International Workshop on, 1988, pp. 485-489.

[23] S. Yasunobu and T. Hasegawa, "Evaluation of an automatic container crane operation system based on predictive fuzzy control," Controltheory and advanced technology, vol. 2, no. 3, pp. 419-432, 1986.

[24] _ - "Predictive fuzzy control and its application for automatic container crane operation system," in Proceedings of the 2nd. IFSA Congress, 1987.

[25] J. Smoczek and J. Szpytko, "Comparision of model predictive, input shaping and feedback control for a lab-scaled overhead crane," in Proceedings of the International Conference on Methods and Models in Automation and Robotics, 2016, pp. 288-293.

[26] K. Graichen, M. Egretzberger, and A. Kugi, "Suboptimal model predictive control of a laboratory crane," IFAC Proceedings Volumes, vol. 43, no. 14, pp. 397-402, 2010.

[27] L. Van den Broeck, M. Diehl, and J. Swevers, "Experimental validation of time optimal MPC on a flexible motion system," in Proceedings of the American Control Conference, pp. 4749-4754.

[28] J. Smoczek and J. Szpytko, "Soft-constrained predictive control for an overhead crane," Journal of KONES Powertrain and Transport, vol. 24, no. 3, 2017

[29] D. Schindele and H. Aschemann, "Fast nonlinear MPC for an overhead travelling crane," IFAC Proceedings Volumes, vol. 44, no. 1, pp. $7963-$ 7968, 2011.

[30] Z. Wu, X. Xia, and B. Zhu, "Model predictive control for improving operational efficiency of overhead cranes," Nonlinear Dynamics, vol. 79, no. 4, pp. 2639-2657, 2015.

[31] Z. Wu and X. Xia, "Energy efficiency of overhead cranes," IFAC Proceedings Volumes, vol. 47, no. 3, pp. 19-24, 2014
[32] A. Khatamianfar and A. V. Savkin, "A new tracking control approach for 3d overhead crane systems using model predictive control," in Proceedings of the European Control Conference, pp. 796-801.

[33] D. Jolevski and O. Bego, "Model predictive control of gantry/bridge crane with anti-sway algorithm," Journal of mechanical science and technology, vol. 29, no. 2, pp. 827-834, 2015.

[34] B. Käpernick and K. Graichen, "Model predictive control of an overhead crane using constraint substitution," in Proceedings of the American Control Conference, 2013, pp. 3973-3978.

[35] H. Chen, Y. Fang, and N. Sun, "A swing constraint guaranteed MPC algorithm for underactuated overhead cranes," IEEE/ASME Transactions on Mechatronics, vol. 21, no. 5, pp. 2543-2555, 2016.

[36] M. Vukov, W. Van Loock, B. Houska, H. J. Ferreau, J. Swevers, and M. Diehl, "Experimental validation of nonlinear MPC on an overhead crane using automatic code generation," in Proceedings of the American Control Conference, 2012, pp. 6264-6269.

[37] W. Singhose, L. Porter, M. Kenison, and E. Kriikku, "Effects of hoisting on the input shaping control of gantry cranes," Control Engineering Practice, vol. 8, no. 10, pp. 1159-1165, 2000

[38] D. Lewis, G. G. Parker, B. Driessen, and R. D. Robinett, "Command shaping control of an operator-in-the-loop boom crane," in Proceedings of the American Control Conference, vol. 5, 1998, pp. 2643-2647.

[39] D. Kim and W. Singhose, "Performance studies of human operators driving double-pendulum bridge cranes," Control Engineering Practice, vol. 18, no. 6, pp. 567-576, 2010.

[40] J. Vaughan, A. Karajgikar, and W. Singhose, "A study of crane operator performance comparing PD-control and input shaping," in Proceedings of the American Control Conference, 2011, pp. 545-550.

[41] L. Ramli, Z. Mohamed, A. M. Abdullahi, H. Jaafar, and I. M. Lazim, "Control strategies for crane systems: A comprehensive review," Mechanical Systems and Signal Processing, vol. 95, pp. 1-23, 2017.

[42] D. Qian and J. Yi, Hierarchical sliding control for under-actuated cranes. Springer, 2015.

[43] M. Faroni, M. Beschi, M. Berenguel, and A. Visioli, "Fast MPC with staircase parametrization of the inputs: Continuous input blocking," in IEEE International Conference on Emerging Technologies and Factory Automation, 2017, pp. 1-8.

[44] L. Wang, Model predictive control system design and implementation using MATLAB®. Springer Science \& Business Media, 2009.

[45] J. A. Rossiter, Model-based predictive control: a practical approach. CRC press, 2003.

[46] C. Mathworks and T. Simscape, "Multibody (tm) getting started guide, 2016," 2016. 